\title{
Plant Cell Reports turns 40
}

\author{
Günther Hahne ${ }^{1} \cdot$ C. Neal Stewart ${ }^{2}$
}

Received: 15 July 2021 / Accepted: 15 July 2021 / Published online: 23 July 2021

(c) The Author(s), under exclusive licence to Springer-Verlag GmbH Germany, part of Springer Nature 2021

The first issue of Plant Cell Reports was published in August 1981. The journal was founded by Olaf Gamborg and Klaus Hahlbrock because: "A journal especially devoted to the rapid publication of short communications has so far been missing in the field of plant cell research (O. Gamborg, K. Hahlbrock, Plant Cell Reports (1981) 1: 1).” It was "designed to be a platform for rapid communication among all scientists with an interest in the physiology, biochemistry, molecular biology, genetics, and structure of higher plant cells, including related aspects of plant breeding and medical/ pharmaceutical sciences (O. Gamborg, K. Hahlbrock, Plant Cell Reports (1981) 1: 1)." At that time, I (G.H.) just started to work on my Ph.D. From my point of view, Plant Cell Reports had always been part of the scientific landscape, all through my professional training and later career. Later, I worked as a postdoc in the Max-Planck-Institut für Züchtungsforschung in Cologne where Klaus Hahlbrock was one of the directors. He was succeeded by Nikolaus Amrhein. A decade later, I (G.H.) had the pleasure to share the Editor-in-Chief responsibility with Oluf Gamborg. Later Editor-in-Chief teams included, successively, Michael Horn and Jang R. Liu.

Plant Cell Reports was created to cater for the needs of "plant cell research," at a time that was at the infancy of plant biotechnology. While "biotechnology" and "plants" were just finding their synergy, Plant Cell Reports soon filled a niche for publishing on the topic. In 1981 the hot plant biotechnology topics included tissue culture, plant regeneration, protoplasts, horizontal gene transfer, haploid cell production, and biosynthesis of important compounds from cultured plant cells. The journal was an outlet for reporting methodologies that were devised, perfected, and extended to more and more species. The published studies were often technology-oriented during the period where the

\footnotetext{
Günther Hahne

plantcellreports@ghahne.fastmail.fm

Weinheim, Germany

2 Knoxville, USA
}

technological foundations of plant biotechnology were laid down. A consistent subject of discussion concerned the socalled "recalcitrant species," which did not easily respond to the then-standard methods and treatments in tissue culture or transformation. Gramineae were especially recalcitrant in this respect: regeneration and, in particular, genetic transformation of cereal crops such as wheat, maize, and rice were considered impossible for quite some time. All this, however, was solved as technology advanced. Arabidopsis was not a prominent topic, but it notably appeared in one article published in 1981 given its role of being one parent in an interspecific"parasexual" hybrid named Arabidobrassica (I.K. Komarnitzky and Yu. Yu. Gleba, Plant Cell Reports (1981) 1: 67-68). The next mention of Arabidopsis in the journal came full 7 years later, in 1988, and concerns its high-efficiency genetic transformation (R. Schmidt and L. Willmitzer, Plant Cell Reports (1988) 7: 583-586).

What did the journal look like? The original idea developed by Gamborg and Hahlbrock was, indeed, quite original. Here again, available technology had been the driving factor: the overreaching goal was rapid communication of new knowledge at a time when that knowledge was created at an unprecedented speed. The initial editorial explained the concept: "As soon as Plant Cell Reports has achieved its proposed rate of bimonthly appearance, camera-ready typescripts will be published within two to ten weeks from the date of acceptance (O. Gamborg, K. Hahlbrock, Plant Cell Reports (1981) 1: 1).“This unheard-of speed of publication was made possible by the submission of camera-ready manuscripts on offset templates. The production of those manuscripts, limited to five pages, put quite a demand on the authors, whose general experience with word processing applications was largely non-existent (these became widely used only several years later). Typos were hard to correct on those templates and often remained in the published text as did the many different fonts used. In addition, authors displayed ingenuity to squeeze a maximum of text and figures into those five pages. Needless to say, this did not compromise the quality of scientific evaluation. What may seem like a somewhat folkloristic touch from today's perspective was 
a highly efficient tool for rapid communication of the latest findings in plant cell research and biotechnology, which from the beginning assured a highly respected place for the new journal among the competing literature. Indeed, a perusal of the authors in the list of papers at the end of this narrative finds the names of many of the top innovators in plant biotechnology.

Forty years later, Plant Cell Reports still enjoys a remarkably high esteem among the scientists interested in plant cell research and biotechnology. Much has remained, e.g., the importance of rapid communication and short publishing times, the widespread scope of topics published in the journal, and publications on non-model species. But also, much has changed. The majority of papers published in Plant Cell Reports are no longer methodology-centered. Papers now provide novel insight into biological mechanisms. Novel insight into fundamental principles does not necessarily require the use of model plants. Plant Cell Reports is an outlet that shows that research on non-model plants, often those cultivated for their agronomic or horticultural virtues, can reveal knowledge that might have been missed if research were restricted to model species. The journal has also aimed to be responsive to the needs of the scientific community. Reviews have become a regular component of the journal's offer. Special issues shine a particularly intense light on hot and timely subjects through a judicious mix of original papers, reviews, and opinion papers. A link to the origins of the journal exists in the form of Focus articles, which provide a short format ideally suited for the rapid publication of ideas or findings that do not need to be developed in much length to make their impact.

The more than 6300 articles published in Plant Cell Reports over the years reflect the evolution of the science in plant science, but also of the publication and citation practices. The 29 articles published in 1981 were cited a total of 816 times (on average, 28 times in 40 years or 0.7 times/year); those 213 papers published in 1998 were cited 6688 times (on average, 31 times in 23 years or 1.3 times/ year), and those 179 published in 2015 were 3628 times (on average 20 times in 6 years or 3.3 times/year). Most recently, in 2020, 131 articles were published and already cited 311 times (on average, 2.4 times in less than 1 year). What also has changed is the way of measuring the impact of a scientific journal. Today, the traditional Impact Factor (4.570 in 2020) is joined by Altmetrics, such as Article Downloads (432,578 in 2020) and Social Media Mentions (3.387 in 2020).

While the challenges of scientific publishing are vastly different now from those in 1981, our mission and objectives remain unchanged: the journal is still devoted to serving the scientific community through the rapid publication of high-quality contributions dealing with new advances and insight into biological mechanisms, as well as exceptionally interesting technological advances in plant cell and molecular biology. The reputation of this journal is entirely the result of the dedication of all the editors and reviewers who have ensured high standards of the published articles throughout the 40 years of its existence and this is the occasion to warmheartedly acknowledge the excellence of their work.

The highest reward, however, for all of us involved in the publication of Plant Cell Reports-both past and present-is the appreciation of our contributors and readers. All available indicators suggest that the journal enjoys the same, if not higher, level of appreciation today as it did at the moment of its launch-and we are highly motivated to keep it that way.

Günther Hahne

C. Neal Stewart Jr.

Editors-in-Chief

\section{Selected papers over the decades}

1. Fraction I protein analysis of parasexual hybrid plants Arabidopsis thaliana + Brassica campestris. Komarnitsky and Gleba (1981) https://doi.org/10.1007/bf002 69275

2. Production of shikonin derivatives by cell suspension cultures of Lithospermum erythrorhizon. Fujita et al. (1981) https://doi.org/10.1007/bf00269273

3. Mitotic protoplasts and their infection with tobacco mosaic virus RNA encapsulated in liposomes. Nagata et al. (1982) https://doi.org/10.1007/bf00272631

4. Agarose plating and a bead type culture technique enable and stimulate development of protoplast-derived colonies in a number of plant species. Shillito et al. (1983) https://doi.org/10.1007/bf00269151

5. Leaf disc transformation of cultivated tomato (L. esculentum) using Agrobacterium tumefaciens. McCormick et al. (1986) https://doi.org/10.1007/bf00269239

6. Stimulation of shoot regeneration in Triticum aestivum and Nicotiana plumbaginifolia Viv. tissue cultures using the ethylene inhibitor $\mathrm{AgNO}_{3}$. Purnhauser et al. (1987) https://doi.org/10.1007/bf00269725

7. Transgenic rice plants produced by electroporationmediated plasmid uptake into protoplasts. Zhang et al. (1988) https://doi.org/10.1007/bf00269517

8. High efficiency Agrobacterium tumefaciens-mediated transformation of Arabidopsis thaliana leaf and cotyledon explants. Schmidt and Willmitzer (1988) https:// doi.org/10.1007/bf00272763

9. Development of the particle inflow gun for DNA delivery to plant cells. Finer et al. (1992) https://doi.org/10. 1007/bf00233358 
10. Transgenic plant production mediated by Agrobacterium in Indica rice. Rashid et al. (1996) https://doi.org/ $10.1007 / \mathrm{bf} 00232216$

11. Roles of the reactive oxygen species-generating peroxidase reactions in plant defense and growth induction. Kawano (2003) https://doi.org/10.1007/s00299-0030591-z

12. Peroxidases have more functions than a Swiss army knife. All and Green (2005) https://doi.org/10.1007/ s00299-005-0972-6

13. Plant tolerance to drought and salinity: stress regulating transcription factors and their functional significance in the cellular transcriptional network. Golldack et al. (2011) https://doi.org/10.1007/s00299-011-10680

14. The interaction between nitrogen availability and auxin, cytokinin, and strigolactone in the control of shoot branching in rice (Oryza sativa L.). Xu et al. (2015) https://doi.org/10.1007/s00299-015-1815-8

15. Efficient targeted multiallelic mutagenesis in tetraploid potato (Solanum tuberosum) by transient CRISPRCas9 expression in protoplasts. Andersson et al. (2016) https://doi.org/10.1007/s00299-016-2062-3
16. Root exudates: from plant to rhizosphere and beyond. Vives-Peris et al. (2019) https://doi.org/10.1007/ s00299-019-02447-5

17. Synergistic antiviral effects against SARS-CoV-2 by plant-based molecules. Prasad et al. (2020) https://doi. org/10.1007/s00299-020-02560-w

18. Dynamics of cell wall structure and related genomic resources for drought tolerance in rice. Ganie and Ahammed (2021) https://doi.org/10.1007/s00299-02002649-2

Until the end of 2021, this collection of articles will also be highlighted on the journal's homepage at https://www. springer.com/journal/299/updates

Publisher's Note Springer Nature remains neutral with regard to jurisdictional claims in published maps and institutional affiliations. 